\title{
Metallic photonic band-gap materials
}

\author{
M. M. Sigalas, C. T. Chan, K. M. Ho, and C. M. Soukoulis \\ Ames Laboratory and Department of Physics and Astronomy, Iowa State University, Ames, Iowa 50011
}

(Received 15 February 1995)

\begin{abstract}
We calculate the transmission and absorption of electromagnetic waves propagating in twodimensional (2D) and 3D periodic metallic photonic band-gap (PBG) structures. For 2D systems, there is substantial difference between the $s$ - and $p$-polarized waves. The $p$-polarized waves exhibit behavior similar to the dielectric PBG's. But, the $s$-polarized waves have a cutoff frequency below which there are no propagating modes. For 3D systems, the results are qualitatively the same for both polarizations but there are important differences related to the topology of the structure. For 3D structures with isolated metallic scatterers (cermet topology), the behavior is similar to that of the dielectric PBG's, while for 3D structures with the metal forming a continuous network (network topology), there is a cutoff frequency below which there are no propagating modes. The systems with the network topology may have some interesting applications for frequencies less than about $1 \mathrm{THz}$ where the absorption can be neglected. We also study the role of the defects in the metallic structures.
\end{abstract}

\section{INTRODUCTION}

Recently, there has been growing interest in the development of easily fabricated photonic band-gap (PBG) materials; ${ }^{1-22}$ these are periodic dielectric materials exhibiting frequency regions where electromagnetic (em) waves cannot propagate. The reason for the interest on PBG materials arises from the possible applications of those materials in several scientific and technical areas such as filters, optical switches, cavities, design of more efficient lasers, etc. ${ }^{1,2}$ Most of the research effort has been concentrated in the development of two-dimensional (2D) and 3D PBG materials consisting of positive and frequency-independent dielectrics ${ }^{1-19}$ because, in that case, one neglect the possible problems related to the absorption. ${ }^{15,20}$

However, there is some more recent work on PBG materials constructed from metals ${ }^{21,22}$ which suggests that those metallic structures may be advantageous in lowfrequency regions where the metals become almost perfect reflectors. In this paper, we study $2 \mathrm{D}$ and $3 \mathrm{D}$ metallic PBG structures; in order to find the possible advantages of metallic PBG materials, we compare their results with the corresponding results for PBG materials constructed with positive and frequency-independent dielectric constants. Also, by changing the scale of the structures, we find the frequency regions where metallic PBG materials can be practically used.

We use the transfer-matrix method (TMM), recently introduced by Pendry and MacKinnon, ${ }^{14}$ to calculate the em transmission through the PBG materials. In the TMM, the total volume of the system is divided into small cells and the fields in each cell are coupled to those in the neighboring cells. Then the transfer matrix can be defined by relating the incident fields on one side of the PBG structure with the outgoing fields on the other side. Using the TMM, the band structure of an infinite periodic system can be calculated, but the main advantage of this method is the calculation of the transmission and reflection coefficients for em waves of various frequencies incident on a finite-thickness slab of the PBG material. In that case, the material is assumed to be periodic in the directions parallel to the interfaces. The TMM has previously been applied in studies of defects in 2D PBG structures, ${ }^{15}$ of PBG materials in which the dielectric constants are complex and frequency dependent, ${ }^{20}$ of $3 \mathrm{D}$ layer-by-layer PBG materials, ${ }^{18}$ and of 2D metallic PBG structures. $^{22}$ In all these examples, the agreement between theoretical predictions and experimental measurements was very good.

We use the following frequency-dependent dielectric constant for the metal:

$$
\epsilon(v)=1-\frac{v_{p}^{2}}{v(v-i \gamma)},
$$

where $v_{p}=3600 \mathrm{THz}$ and $\gamma=340 \mathrm{THz}$ are the plasma frequency and the absorption coefficient. From the previous equation, it turns out that the conductivity is ${ }^{23}$ $\sigma=v_{p}^{2} \gamma / 2\left(\gamma^{2}+v^{2}\right)$. For frequencies smaller than about $100 \mathrm{THz}, \sigma$ can be practically assumed independent of frequency and equal to $0.22 \times 10^{5}(\Omega \mathrm{cm})^{-1}$ which is very close to the $\sigma$ of Ti. However, the conclusions are similar for any other metal. The skin depth is ${ }^{23}$ $\delta=c(\mu v \sigma)^{-1 / 2} / 2 \pi$ where $c$ is the velocity of light aid $\mu$ is the magnetic permeability which is 1 in our case. So for $v=100$ and $10 \mathrm{THz}$, the skin depth is 0.035 and 0.11 $\mu \mathrm{m}$, respectively.

\section{2D STRUCTURES}

In this section, we study 2D systems consisting of infinitely long metallic cylinders parallel to the $z$ axis, embedded in air and forming a square lattice with lattice constant $a$; the systems have finite thickness $L$ along the $y$ axis and they are infinite along the $x$ axis (similar systems with dielectric cylinders have been studied in Refs. 7, 9, and 12). The $k$ vectors, of the incident em waves form an 
angle $\theta$ with the $y$ axis and their $E$ field is either parallel or perpendicular ( $s$ or $p$ polarization) to the $z$ axis. Each unit cell is divided into $20 \times 20$ cells resulting in a convergence of better than $3 \%$.

Figure 1 shows the transmission and absorption of $s$ polarized waves for a system consisting of metallic cylinders with filling ratio $f=0.1, a=1.27 \mu \mathrm{m}$, and $L=2 a$. For $\theta=0^{\circ}$ (solid lines in Fig. 1), there are two gaps in the transmission separated by a sharp peak which is located at around $125 \mathrm{THz}$. The lowest gap extends from zero up to a cutoff frequency $v_{c}=125 \mathrm{THz}$ and it is very sharp since there is $-23 \mathrm{~dB}$ per unit cell drop in the transmission at $50 \mathrm{THz}$. This cutoff frequency is an important feature of $s$-polarized waves propagating in $2 \mathrm{D}$ metallic PBG structures which distinguishes them from the corresponding case of 2D dielectric PBG structures. The second weak gap (from around 125 to $200 \mathrm{THz}$ ) is less sharp with $-9 \mathrm{~dB}$ per unit cell drop in the transmission at $160 \mathrm{THz}$. The absorption [Fig. 1(b)] increases almost linearly with the frequency except for a sharp peak at $125 \mathrm{THz}$. Note that there is a peak in the transmission at around the same frequency. This is the result of the fact that the wave can actually propagate through the whole system while for frequencies inside the gaps the wave is actually reflected, so one expects the absorption to be smaller in the frequencies where the gaps appear.
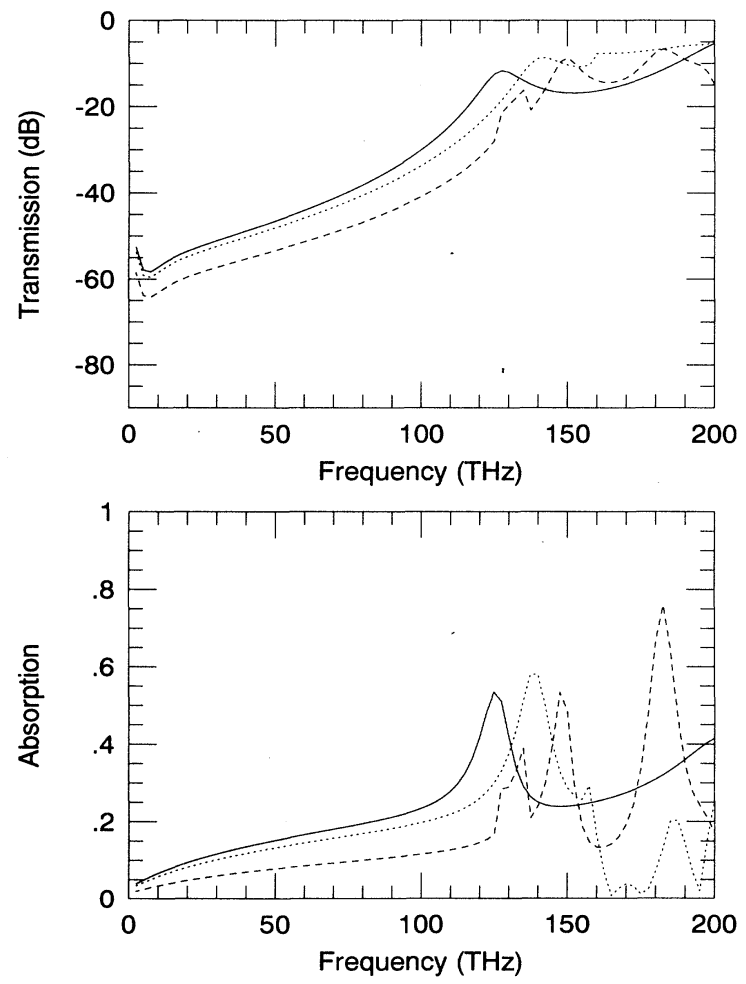

FIG. 1. Transmission and absorption for s-polarized em waves propagating in a 2D square lattice consisting of metallic cylinders with filling ratio $f=0.1$ embedded in air; the lattice constant is $a=1.27 \mu \mathrm{m}$ and the thickness is $L=2 a$. Solid, dotted, and dashed lines correspond to incident angles $\theta=0^{\circ}, 30^{\circ}$, and $60^{\circ}$, respectively.
Increasing the incident angle, the second gap tends to disappear while the first gap survives and actually becomes wider; in particular, the cutoff frequency $v_{c}$ is 140 and $150 \mathrm{THz}$ for $\theta=30^{\circ}$ and $40^{\circ}$, respectively. Also the absorption [Fig. 1(b)] becomes smaller (especially at low frequencies) as the incident angle increases.

The behavior of the transmission is different for the $p$ polarization. For $\theta=0^{\circ}$ [solid line in Fig. 2(a)], there is only a small drop in the transmission at around $115 \mathrm{THz}$; this drop tends to disappear as the incident angle increases while other sharper drops appear at higher frequencies [see Fig. 2(a)]. In other words, the transmission profiles for the $p$-polarized em waves propagating in metallic PBG structures are similar to the profiles of em waves propagating in PBG materials with positive and frequency-independent dielectric constants. The absorption increases almost linearly as a function of frequency, and in general it increases as the incident angle increases [Fig. 2(b)].

In order to explain the previous results, we make the plausible assumption that the cylinders form infinitely long metallic plates parallel to the $x, y$ plane with small thickness separated by $d$. For $s$-polarized waves, the previous assumption leads to a simple waveguide model with dimension $d$. In that case, the allowed propagating modes are given by the relation

$$
v_{m}=\frac{m c}{2 d \sqrt{\epsilon_{0}}},
$$

where $m=1,2,3, \ldots, c$ is the velocity of light in air and
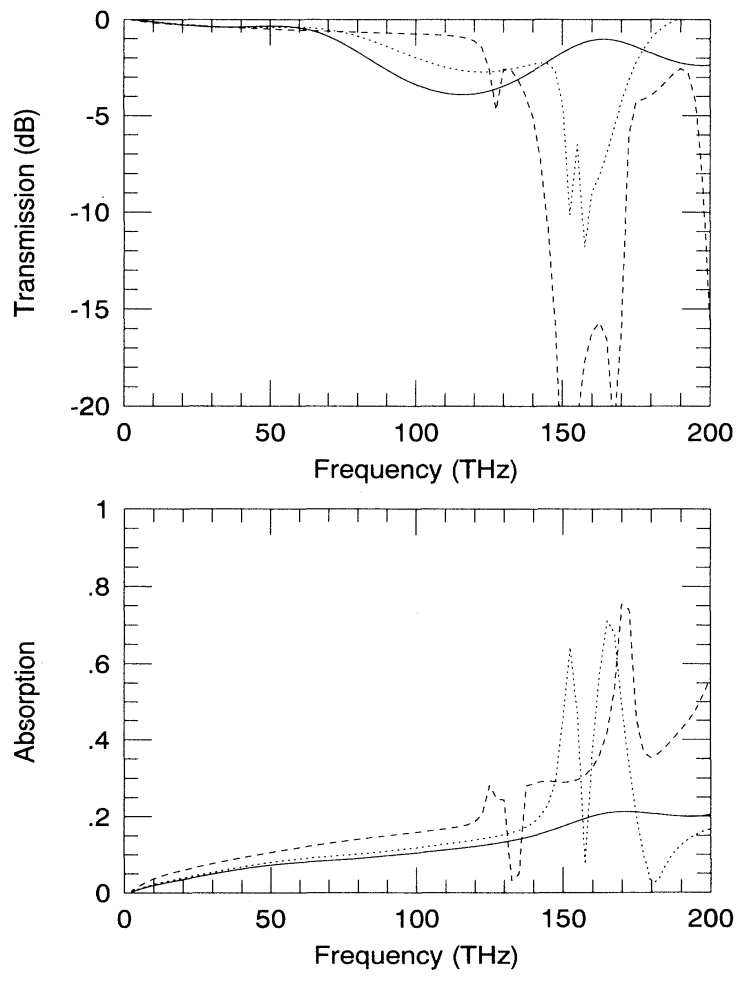

FIG. 2. The same as in Fig. 1 for $p$-polarized em waves. 
$\epsilon_{0}$ is the dielectric constant for the material which is between the metallic plates. So for $\epsilon_{0}=1$ and assuming that $d$ is roughly equal to the lattice constant, $a=1.27 \mu \mathrm{m}$, the lowest allowed mode is $v_{1}=118 \mathrm{THz}$ which is in good agreement with the cutoff frequency of $125 \mathrm{THz}$ for the $s$ polarization (Fig. 1). Also the previous formula [Eq. (2)] suggests that the cutoff frequency depends on the dielectric constant of the surrounding medium ( $v_{c}$ depends on $\epsilon_{0}^{1 / 2}$ ) which is also in agreement with the TMM results. For the $p$ polarization, the waveguide model does not apply and therefore no cutoff frequency is obtained in agreement with the TMM results (Fig. 2). Note that the results are qualitatively similar to those obtained from the electromagnetic theory of gratings. ${ }^{24}$ However, in the case of gratings, one is dealing with just one layer of perfectly arranged metallic cylinders while in the present case, we are studying cases with more than one layer as well as cases in which defects have been introduced in an initially periodic system.

For $s$-polarized waves, by decreasing the filling ratio $f$, the cutoff frequency becomes smaller while the transmission increases for all frequencies; in particular, for $a=1.27 \mu \mathrm{m}, \theta=0^{\circ}$, and $L=2 a, v_{c}$ is 100 and $125 \mathrm{THz}$ for $f=0.03$ and 0.10 , respectively. By decreasing the filling ratio, the distance $d$ in the waveguide model described previously [Eq. (2)] increases. So the cutoff frequency decreases, in accordance with the TMM results. Keeping the filling ratio constant, the transmission $T$ decreases almost exponentially with thickness for all the frequencies inside the gaps but the transmission at the cutoff frequency remains almost the same.

Figure 3 shows the transmission and the absorption of $s$-polarized waves as a function of the dimensionless frequency $v a / c$ for $L=2 a, f=0.1$, and $\theta=0^{\circ}$. In contrast to the dielectric PBG materials, the results change as we scale the dimensions of the structure. The dimensionless cutoff frequency $v_{c} a / c$ is $0.42,0.54$, and 0.55 for $a=0.127,1.27$, and $12.7 \mu \mathrm{m}$, respectively. This indicates that there is a convergence of the dimensionless cutoff frequency to a constant value, so the cutoff frequency in the microwave and millimeter regions can be predicted. Comparison of the transmission for $a=1.27$ and $12.7 \mu \mathrm{m}$ [dotted and dashed lines in Fig. 3(a)] shows that the peak in the transmission at the cutoff frequency becomes sharper as the lattice constant increases. The absorption [Fig. 3(b)] becomes smaller as the lattice constant increases; in particular, the absorption at $v a / c=0.2$ is $0.31,0.13$, and 0.04 for $a=0.127,1.27$, and $12.7 \mu \mathrm{m}$, respectively. For a constant value of $v a / c$, the ratio of the skin depth to the lattice constant $a$ is inversely proportional to the square root of $a$ (this is correct for frequencies less than $100 \mathrm{THz}$ ). This means that, by increasing the lattice constant, the part of the wave which penetrates into the metal (this part is responsible for the absorption) becomes smaller. So the absorption must become smaller as the lattice constant increases and eventually it will be negligible in the microwave and millimeter wave regions. Also, due to the fact that the penetration of the wave into the metal becomes smaller on increasing the lattice constant, the dimension $d$ of the waveguide
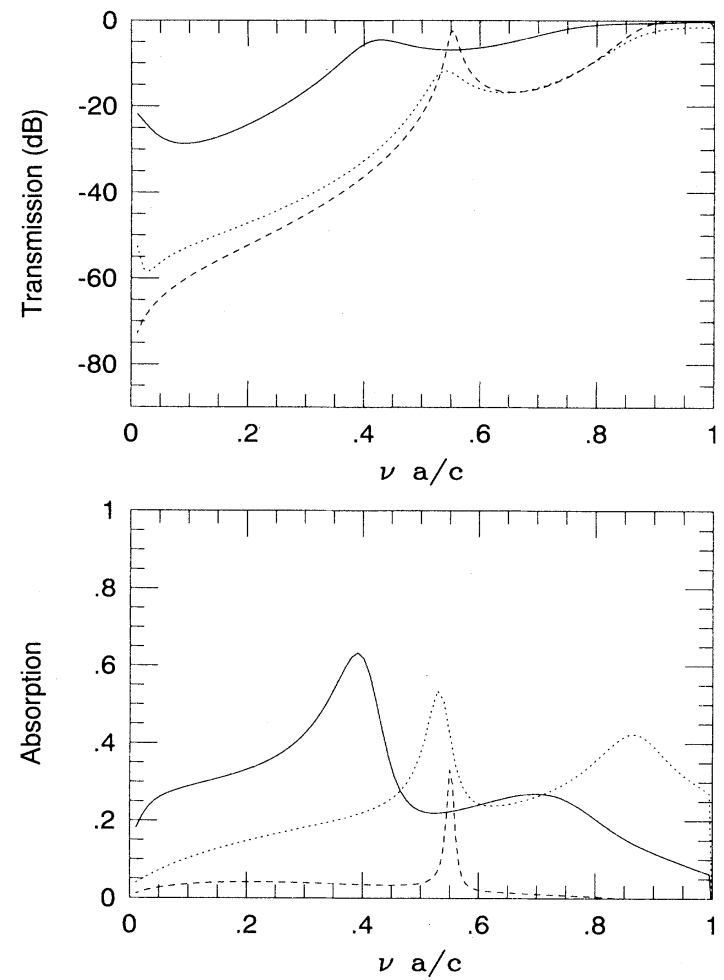

FIG. 3. Transmission and absorption of $s$-polarized em waves propagating in a system similar to the one described in Fig. 1. Solid, dotted, and dashed lines correspond to $a=0.127,1.27$, and $12.7 \mu \mathrm{m}$, respectively; $\theta=0^{\circ}$.

model [Eq. (2)] decreases and the dimensionless cutoff frequently increases in accordance with the TMM results.

In the following part of this section, we study how the transmission and absorption of $s$-polarized waves propagating in the periodic $2 \mathrm{D}$ systems described previously change on the introduction of defects in those structures. A supercell with a size of $3 a$ along the $x$ axis has been used and periodic boundary conditions are imposed at the edges of the supercell along the $x$ axis; the systems are finite along the $y$ axis with thickness $L=3 a ; a=1.27$ $\mu \mathrm{m}, f=0.1$ and $k$ is along the $y$ axis. Two kinds of defects are introduced. First, by adding one cylinder in the system (dotted lines in Fig. 4); in that case the transmission and absorption change slightly from the corresponding values of the periodic case (compare solid and dotted lines in Fig. 4). Second, by changing the radius $r_{d}$ of one of the cylinders; the ratio $r_{d} / r$ (where $r$ is the radius in the periodic case) gives the amount of discrepancy from the periodicity. For $r_{d} / r=0$ (this corresponds to a case where one of the cylinders is removed; dashed lines in Fig. 4) there is a peak in the transmission due to the defect at $v=87 \mathrm{THz}$. The quality factor is defined as $Q=v_{d} /\left(v_{+}-v_{-}\right) ; v_{d}$ is the frequency where the peak appears and $v_{+}$and $v_{-}$are the frequencies above and below $v_{d}$ where the transmission in $3 \mathrm{~dB}$ smaller than that at $v_{d}$. For $r_{d} / r=0$, by increasing the lattice con- 

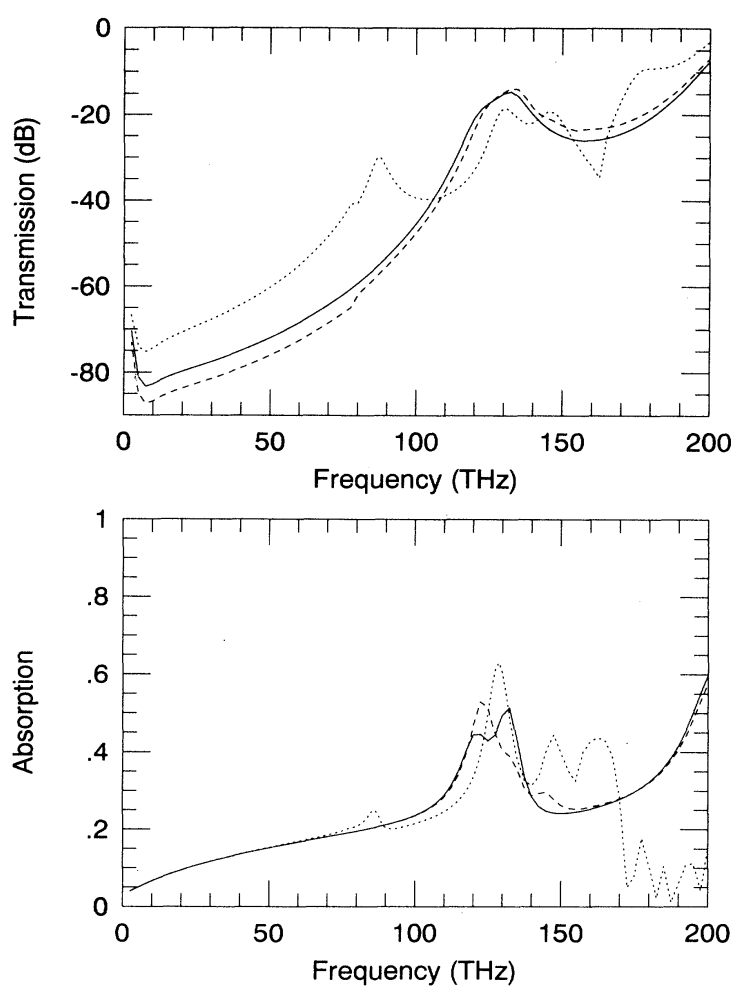

FIG. 4. Transmission and absorption for s-polarized em waves propagating in a $2 \mathrm{D}$ square lattice consisting of metallic cylinders with filling ratio $f=0.1$ embedded in air; the lattice constant is $a=1.27 \mu \mathrm{m}$, the thickness is $L=3 a$, and $\theta=0^{\circ}$. A supercell of width $3 a$ has been used with periodic boundary conditions at the edges of the supercell. Results for the cases where one cylinder is added (dashed), one cylinder is removed (dotted), and the perfect lattice (solid) are shown.

stant $a, Q$ and the transmission at the top of the peak increase, while the dimensionless frequency at the peak does not change. In particular, for $a=6.35$ and $12.7 \mu \mathrm{m}$, the transmission at the top of the peak is -26 and -20 $\mathrm{dB}$, respectively, $Q$ is 39 and 34 , respectively, while the dimensionless frequency of the defect is 0.38 in both cases. Finally, we find that $Q$ decreases sharply and $v_{d}$ increases as $r_{d} / r$ increases. In particular, for $a=1.28$ $\mu \mathrm{m}$ and $f=0.1, Q=2$ and $\nu_{d}=91$ THz when $r_{d} / r=0.05$ while for $r_{d} / r=0.1$ there is only a small shoulder instead of a peak at $100 \mathrm{THz}$.

\section{3D STRUCTURES}

We study first 3D systems consisting of isolated metallic scatterers embedded in air (cement topology 25,26 ). Figure 5 shows the transmission and absorption of em waves propagating in a simple cubic (sc) lattice consisting of metallic spheres with filling ratio $f=0.03$. The system is infinite along the $x$ and $y$ directions while its thickness along the $z$ axis is $L=4 a$ and the incident waves have $\mathbf{k}$ along the $z$ axis. The results for both polarizations are the same due to the symmetry of the lattice. For the
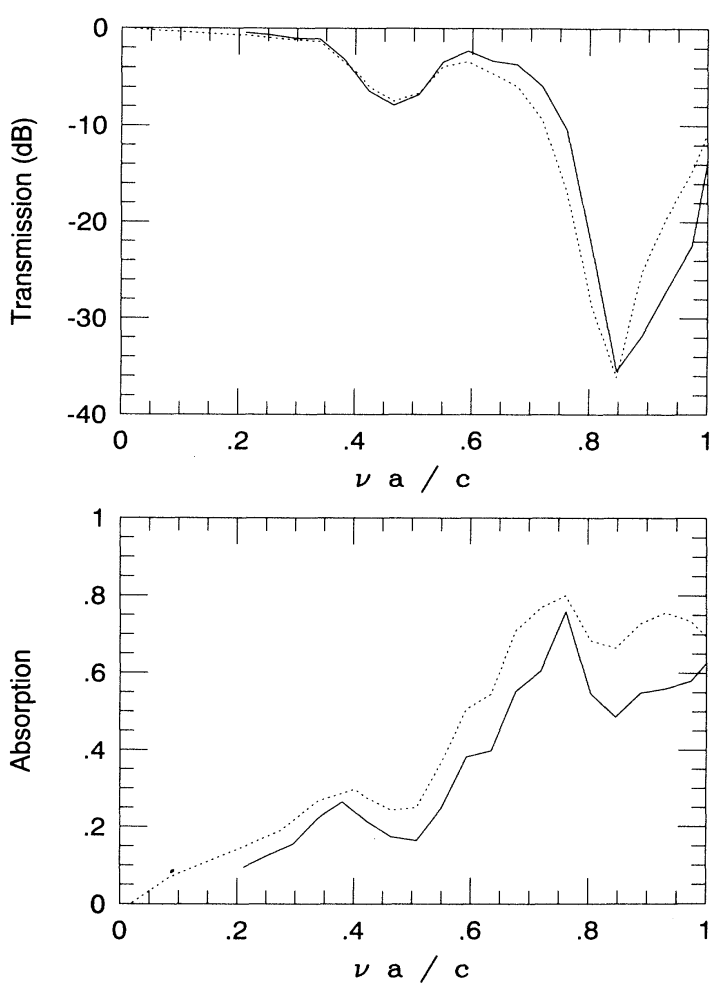

FIG. 5. Transmission and absorption vs the dimensionless frequency $v a / c$ for em waves propagating in a 3D sc lattice consisting of metallic spheres with $f=0.03, L=4 a$, and $\theta=0^{\circ}$. Solid and doted lines correspond to $a=1.27$ and $12.7 \mu \mathrm{m}$.

present as well for all the following cases, each unit cell is divided into $10 \times 10 \times 10$ cells, which gives a convergence of better than $5 \%$ for the periodic cases and better than $15 \%$ for the defect cases. There are two drops in the transmission [Fig. 5(a)]; the first one at around $v a / c=0.45$ and the second (and sharper one) at around 0.85 . $\mathbf{k}$ parallel to the $z$ axis corresponds to the $\Gamma-X$ direction in $k$ space; in that case we expect the first gap to appear at the edge of the zone (at the $X$ point) for $v a / c$ about 0.5 which is slightly higher than the frequency where the first drop in the transmission appears in that direction [Fig. 5(a)]. Due to the small filling ratio, there is no full band gap since the gaps in different directions do not overlap. We find similar results for fcc, bcc, and diamond structures with isolated metallic spheres or cubes. So, for the cases where the metal forms isolated scatterers, the results are similar to those for the dielectric PBG materials. The present results for the isolated metallic scatterers are in agreement with the results of a recent work ${ }^{27}$ in which monolayers consisting of metallic spheres with radius between 10 and $100 \mathrm{~nm}$ were studied.

From Fig. 5, we can also find what happens as we scale the dimensions of the system, assuming that the filling ratio of the system remains the same. By comparing the results for two lattice constants $a=1.27$ and $12.7 \mu \mathrm{m}$ (dotted and solid lines in Fig. 5), we find that the transmission is almost the same for both cases as long as $v a / c$ is less 
than about 0.55 . For higher frequencies, the transmission of the $a=12.7 \mu \mathrm{m}$ case is slightly higher than the one for the $a=1.27 \mu \mathrm{m}$ case. The absorption, however, is always smaller for the $a=12.7 \mu \mathrm{m}$ case. By increasing the lattice constant, the frequency decreases and the absolute value of the dielectric constant of the metal [see Eq. (1)] becomes larger; this means that the metal reflects more (and absorbs less) of the power. This is the reason that the absorption decreases as the lattice constant increases. Also, for any lattice constant, there is a drop in the absorption at frequencies where there is drop in the transmission as a result of the fact that only the first few layers of the material contribute to the absorption.

We now turn to the more interesting case of structures in which the metal forms a network; we shall refer to this case as a network topology. ${ }^{25,26}$ Figure 6 shows the transmission and absorption for a system consisting of metallic tetragonal rods connecting nearest neighbors in a sc lattice (see Ref. 28 for more details about this structure). The system is infinite along the $x$ and $y$ directions while its thickness along the $z$ axis is $L=4 a$; the lattice constant is $a=1.27 \mu \mathrm{m}$ and the filling ratio is $f=0.03$. For incident waves along the $z$ axis (in that case, the result are identical for both polarizations), there is a sharp drop of the transmission from zero up to a cutoff frequency $v_{c}=105 \mathrm{THz}$; the drop at $v=30 \mathrm{THz}$ is $15 \mathrm{~dB}$ per unit cell. There is also a smaller drop ( $4.5 \mathrm{~dB}$ per unit cell) of
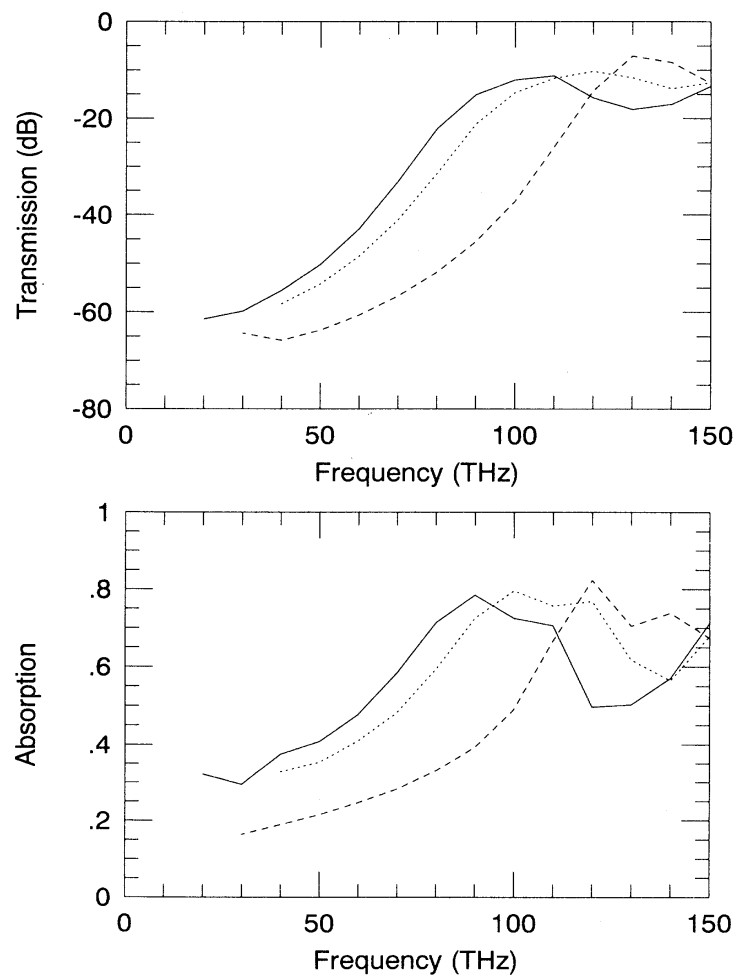

FIG. 6. Transmission and absorption for s-polarized em waves propagating in a $3 \mathrm{D}$ sc lattice consisting of metallic tetragonal rods connecting nearest neighbors with $f=0.03$ and $L=4 a ; a=1.27 \mu \mathrm{m}$. Solid, dotted, and dashed lines correspond to $\theta=0^{\circ}, 30^{\circ}$, and $60^{\circ}$, respectively. the transmission between 110 and $160 \mathrm{THz}$. By increasing the incident angle, the cutoff frequency moves to higher frequencies $\left(\nu_{c}\right.$ is 120 and $130 \mathrm{THz}$ for $\theta=30^{\circ}$ and $60^{\circ}$, respectively); so the first (and sharper) gap survives for any incident angle but the second (and smaller) disappears, on increasing the incident angle. As in the case of isolated metallic scatterers, the absorption increases as the frequency increases and it has maxima at the frequencies where the transmission has a maximum. But the absorption is in general higher for the case of the network topology [compare Figs. 5(b) and 6(b)]. Since the skin depth for frequencies around $100 \mathrm{THz}$ is much smaller than the thickness of the metal, we can assume that the absorption is proportional to the surface area of the metal. But, for the same filling ratio, the surface of the metal is higher in the network topology, so the absorption will be higher for the network topology. As in the case of $s$ polarized em waves in a $2 \mathrm{D}$ system, we can use the same waveguide model to predict the cutoff frequency, but in contrast with the $2 \mathrm{D}$ case the waveguide in the $3 \mathrm{D}$ case is bounded for both polarizations, so we expect the same cutoff frequency for both of them; the predicted cutoff frequency [Eq. (2)] is $118 \mathrm{THz}$ for $d=1.27 \mu \mathrm{m}$ which is very close to the cutoff frequency found by the TMM $\left(\nu_{c}=105 \mathrm{THz}\right)$.

Figure 7 shows the transmission and absorption as a function of the dimensionless frequency $v a / c$, for metal-
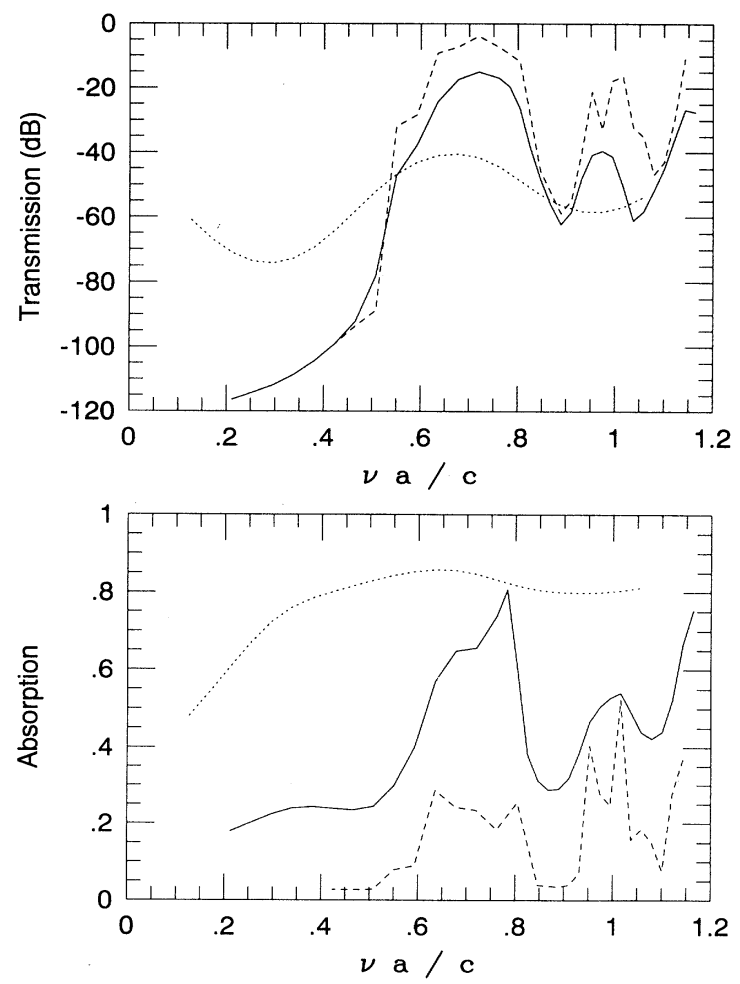

FIG. 7. Transmission and absorption vs the dimensionless frequency $v a / c$ for em waves propagating in a diamond lattice consisting of metallic cylinders connecting nearest neighbors with $f=0.04, L=4 a$, and $\theta=0^{\circ}$. Solid, dotted, and dashed lines correspond to $a=1.27,12.7$, and $127 \mu \mathrm{m}$, respectively. 
lic circular rods connecting nearest neighbors in a diamond structure (more details about this structure can be found in Ref. 4); the filling ratio is $f=0.04$, the thickness of the system is $L=4 a$, and the incident angle is $\theta=0^{\circ}$. For $a=1.27 \mu \mathrm{m}$ there is a peak of the transmission at around $v a / c=0.7$ which separates two relatively small gaps. By increasing the lattice constant the transmission at the peak increases sharply; in particular, the transmission at $v a / c=0.7$ is $-40,-16$, and $-4 \mathrm{~dB}$ for $a=1.27$, 12.7 , and $127 \mu \mathrm{m}$. A peak of the transmission inside the second (higher) gap appears as the lattice constant increases; but that gap does not survive as the incident angle increases, in contrast with the first gap which survives for any incident angle. The absorption has the same behavior as in the sc case with network topology, but it is in general higher for the diamond case [compare the solid lines in Figs. 6(b) and 7(b)]. This is due to the higher surface area of the metal in the diamond case. However, as the lattice constant increases, the absorption becomes smaller; the absorption at $v a / c=0.5$ is $0.83,0.24$, and 0.03 for $a=1.27,12.7$, and $127 \mu \mathrm{m}$; so we expect that the absorption will be negligible in the microwave and millimeter regions. The cutoff frequency for the diamond case is different from the sc case $\left(\nu_{c} a / c=0.44\right.$ and 0.7 for sc and diamond, respectively). Using a similar waveguide model as in the sc case and assuming that the size of the waveguide is given by the distance between the point $\left(\frac{1}{4}, \frac{3}{4}, \frac{3}{4}\right) a$ and the $z=0$ plane (which is $3 a / 4$ ), we find that the predicted cutoff frequency is 0.67 , which is very close to the cutoff frequency of 0.60 calculated from the TMM.

In general, 3D metallic PBG structures with network topology exhibit a cutoff frequency for both polarizations below which there are no propagating modes. This feature puts them in the same category with the $s$ polarized waves propagating in 2D metallic PBG structures. But the features of 3D metallic PBG structures with isolated metallic scatterers are similar to the features of the dielectric PBG structures as well as to the features of $p$-polarized waves in 2D metallic PBG structures (for all the cases in this category there is no cutoff frequency).

We also studied the effect of the introduction of defects in a periodic lattice. We use a sc lattice consisting of metallic tetragonal rods connecting nearest neighbors (network topology) with $f=0.03$ and $a=1.27 \mu \mathrm{m}$, and the surrounding medium is air. A supercell has been used with width $2 a$ along the $x$ and $y$ axes and periodic boundary conditions are imposed at the edges of the supercell; the system is finite along the $z$ axis with thickness $L=3 a$. A defect is introduced by removing part of the metalwhich is included in a sphere of radius $r_{d}$ centered at one of the crossing points of the rods in the second layer. Figure 8 shows the transmission and absorption for such a defect structure and incident waves with $\mathbf{k}$ parallel to the $z$ axis; $a=1.27 \mu \mathrm{m}$. Once again the results are identical for both polarizations due to the symmetry of the structure. For $r_{d} / a=0.15$, a small peak in the transmission appears at around $31 \mathrm{THz}$; the quality factor is very small $(Q=3)$ and the transmission at the top of the peak
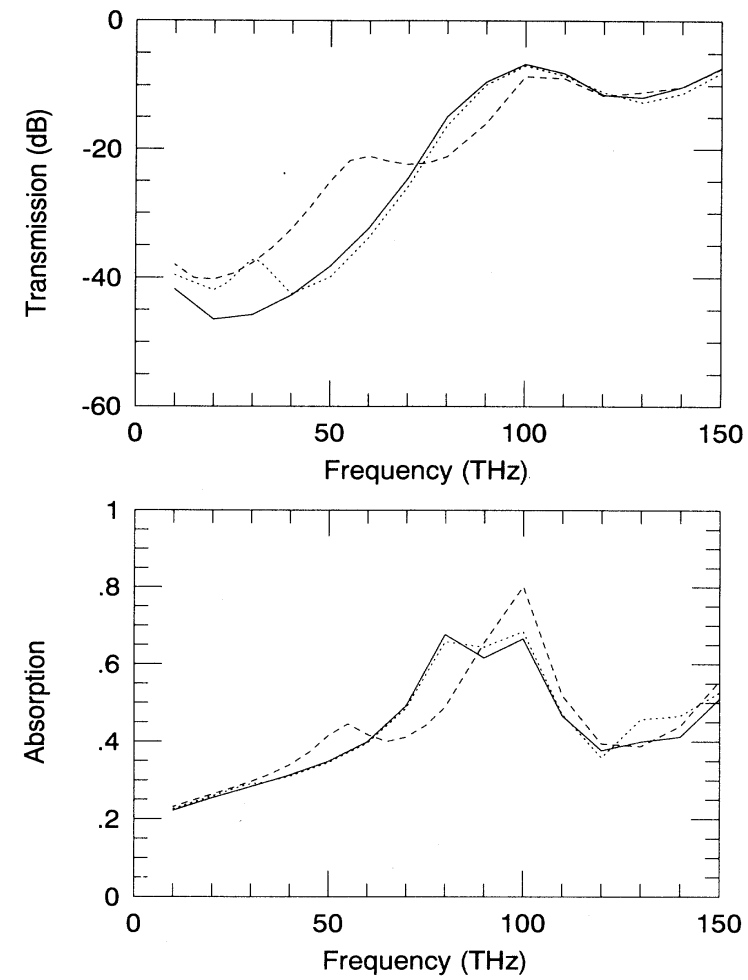

FIG. 8. Transmission and absorption for em waves propagating in a $3 \mathrm{D}$ sc lattice consisting of metallic tetragonal rods connecting nearest neighbors with $f=0.03, L=3 a$, and $\theta=0^{\circ}$. A supercell of width $2 a$ has been used with periodic boundary conditions at the edges of the supercell. The part of the metal which is included in a sphere with the center at one of the crossing points of the rods at the second layer and radius $r_{d}$ has been removed. Solid, dotted, and dashed lines correspond to $t_{d} / a=0,0.15$, and 0.5 , respectively.

is also small $(-37.2 \mathrm{~dB})$. Apart from the frequency region around the defect, the transmissions of the defect and the periodic structures are almost the same [compare the dotted and solid lines in Fig. 8(a)]. For $r_{d} / a=0.5$ (dashed line in Fig. 8), there is a peak in the transmission at higher frequency $(60 \mathrm{THz})$ which is even wider and with higher transmission at the top of the peak. So one can adjust the frequency of the defect inside the gap by just changing the volume of the removed metal; the higher the amount of the removed metal, the higher is the frequency where the defect peak appears. Studies in dielectric PBG materials ${ }^{10}$ have shown that a defect band emerges from the lower edge of the gap and approaches the upper edge of the gap as the volume of the removed dielectric material is increased. The behavior of the defect band is similar in the present case, despite the fact that the lower edge of the gap is actually at zero frequency. The absorption for $r_{d} / a=0.15$ is almost the same as the absorption in the periodic case except for a small maximum at the frequency where the defect peak appears (hardly noticed on comparing dotted and solid lines in Fig. 8). However, the differences in the absorption be- 
tween the $r_{d} / a=0.5$ and the periodic cases (compare dashed and solid lines in Fig. 8) are more obvious the more prominent the peak of the absorption in the $r_{d} / a=0.5$ case at the frequency where the defect appears (60 THz); since the light is trapped around the defect region, one actually expects that the absorption will be higher in that case.

Figure 9 shows the transmission and absorption as a function of the dimensionless frequency $v a / c$ for a defect structure (similar to the one described in the previous paragraph) with $r_{d} / a=0.15$. As we mentioned earlier, the results change as we scale the dimensions of the structure due to the frequency dependence of the dielectric constant [Eq. (1)]. By increasing the lattice constant the transmission at the top of the defect peak, $T_{d}$, and the $Q$ factor increase by orders of magnitude; in particular, $T_{d}=-37,-24$, and $-9 \mathrm{~dB}$ while $Q=3,29$, and 137 for $a=1.27,12.7$, and $127 \mu \mathrm{m}$. However, the dimensionless frequency of the defect, $v_{d} a / c$, increases slightly, reaching a constant value at high lattice constants $\left(v_{d} a / c\right.$ $=0.1312,0.1367$, and 0.1369 for $a=1.27,12.7$, and 127 $\mu \mathrm{m})$. The absorption at $v_{d} a / c$, on the other hand, exhibits more peculiar behavior. In general [see Fig. 9(b)], there is a peak in the absorption exactly at $v_{d} a / c$ (although this peak is hardly noticeable for $a=1.27 \mu \mathrm{m}$ ) which becomes sharper as the lattice constant increases. As we mentioned earlier (see Figs. 3 and 7), for the
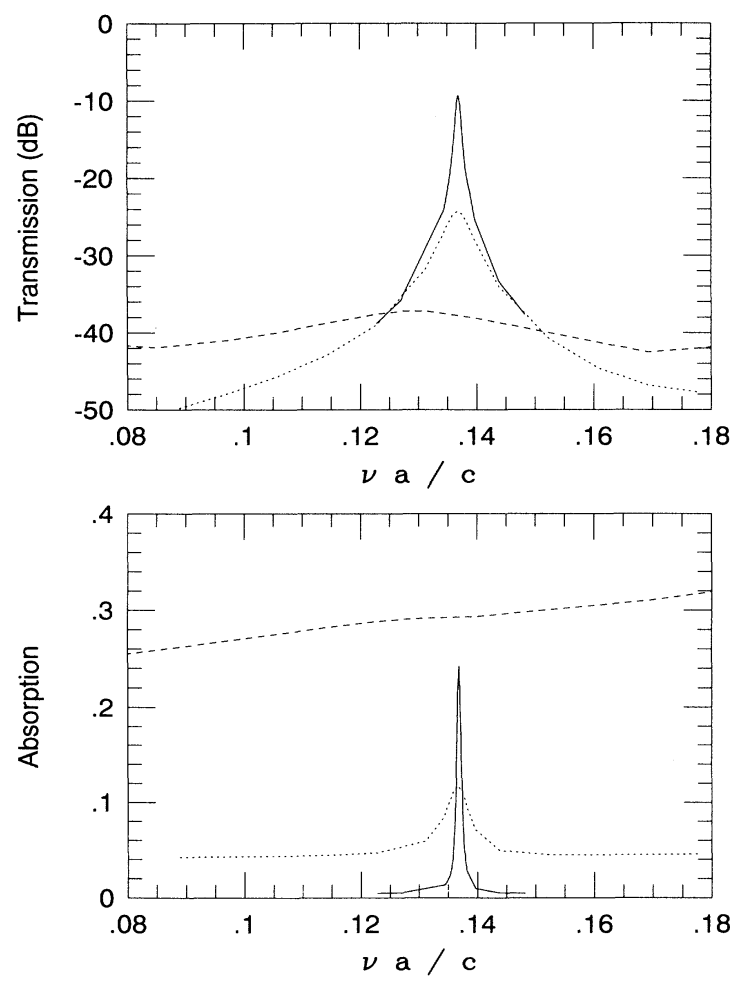

FIG. 9. Transmission and absorption for em waves propagating in a defect structure similar to the one described in Fig. 8 with $r_{d} / a=0.15$. Solid, dotted, and dashed lines correspond to $a=1.27,12.7$, and $127 \mu \mathrm{m}$, respectively. periodic cases, by increasing the lattice constant the overall absorption decreases. Similarly, in the present case, for frequencies well above or below the defect frequency, the overall absorption (we call it background absorption) decreases as the lattice constant increases. At the defect frequency, however, the wave becomes more localized as the lattice constant increases, because the metal becomes a better reflector, so the transmission and absorption peaks become sharper.

\section{CONCLUSIONS}

Using the transfer matrix method, we have calculated the transmission and absorption of em waves propagating in photonic band-gap structures constructed from metals.

For 2D systems consisting of metallic cylinders, there is considerable difference between the two polarizations. For $p$-polarized waves, the results are qualitatively similar to those in dielectric PBG systems. Propagating modes are interrupted by band gaps appearing close to the edges of the Brillouin zone. On the other hand, for $s$-polarized waves, there is a cutoff frequency $v_{c}$. There are no propagating modes for frequencies between zero and $v_{c}$, so the transmission has a very sharp drop in that frequency range. Above $v_{c}$, there is the usual behavior of bands interrupted by gaps.

For 3D metallic PBG structures, the results are very sensitive on the topology of the structure. Systems with isolated metallic scatterers (cermet topology) exhibit similar behavior to that of dielectric PBG materials. But for metallic scatterers forming a continuous network (network topology), there are no propagating modes for frequencies smaller than a cutoff frequency for both polarizations and for any incident angle. Note that for dielectric PBG materials there is no cutoff frequency for both types of the topology. We have shown that this behavior, in both $2 \mathrm{D}$ and $3 \mathrm{D}$ cases, can be explained using a simple waveguide model where the $\nu_{c}$ is predicted with good accuracy. This cutoff frequency is well below the plasma frequency and it is related to the structure of the system.

In all the periodic cases studied, the absorption can be largely neglected for metallic PBG structures with lattice constants $a$ less than about $100 \mu \mathrm{m}$, which correspond to frequencies below about $1 \mathrm{THz}$. So, for frequencies less than about $1 \mathrm{THz}$, wide stop-bands filters constructed from periodic metallic PBG materials can be used as alternatives to similar filters constructed from dielectric PBG materials.

By breaking the connections in the $3 \mathrm{D}$ metallic networks, defect states appear below the cutoff frequency, resulting in a peak in the transmission. The smaller the volume of the removed metal is, the smaller is the frequency where the defect peak appears. As we explain in the next paragraph, this is a very interesting feature of the metallic PBG structure which, in connection with the fact that the filling ratio of the metal can be less than 0.01 , can be used in the construction of narrow bandpass filters smaller in size than those constructed from dielectric PBG materials. By increasing the lattice constant, the $Q$ factor and the transmission at the defect peak increase by orders of magnitude while the dimensionless de- 
fect frequency remains almost constant. The absorption at the frequency where the defect peak appears increases as the lattice constant increases, an effect which may create problems in some of the possible applications.

An important advantage of metallic PBG structures is that they could be smaller in size and lighter than the corresponding dielectric PBG materials. We can see that in the following example. A narrow bandpass filter operating at $323 \mathrm{GHz}$ can be constructed using a metallic PBG material with a defect structure similar to the one described in Fig. 9; this metallic structure would have lattice constant $a=127 \mu \mathrm{m}$ (see solid lines in Fig. 9) and the filling ratio of the metal is less than 0.03 . For a similar bandpass filter constructed from a dielectric PBG material, one needs a band gap at around $323 \mathrm{GHz}$; assuming that the midgap frequency of a dielectric PBG material is given by $v a / c \equiv 1$, the lattice constant of the dielectric PBG would be around $929 \mu \mathrm{m}$ and the filling ratio of the dielectric materials would be higher than about 0.1 . So the dielectric PBG material would be more than seven times larger and heavier than the corresponding metallic
PBG material. Our calculations show that similar metallic PBG materials can be constructed with filling ratios of the metal less than 0.01 , making them even more attractive for applications where the size or weight is a consideration. However, one has to keep in mind that, in the metallic case, there is also a considerable amount of absorption (see the solid lines in Fig. 9) which could be completely neglected in the dielectric case. By decreasing $\gamma$ in Eq. (1) (or, equivalently, by increasing the conductivity of the metal, $\sigma$ ), the absorption becomes smaller. This means that the problem due to the absorption can be avoided by using a superconductor instead of a metal.

\section{ACKNOWLEDGMENTS}

This work was made possible in part by the Scalable Computing Laboratory which is funded by Iowa State University and Ames Laboratory. Ames Laboratory is operated by the U.S. Department of Energy by Iowa State University under Contract No. W-7405-Eng-82.
${ }^{1}$ See Development and Applications of Materials Exhibiting Photonic Band Gaps, special issue of J. Opt. Soc. Am. B 10, 208 (1993).

${ }^{2}$ See Photonic Band Gaps and Localization, Vol. 308 of NATO Advanced Science Institute Series B: Physics, edited by M. Soukoulis (Plenum, New York, 1993).

${ }^{3}$ K. M. Ho, C. T. Chan, and C. M. Soukoulis, Phys. Rev. Lett. 65, 3152 (1990).

${ }^{4}$ C. T. Chan, K. M. Ho, and C. M. Soukoulis, Europhys. Lett. 16, 563 (1991).

${ }^{5}$ E. Yablonovitch, T. J. Gmitter, and K. M. Leung, Phys. Rev. Lett. 67, 2295 (1991).

${ }^{6}$ H. S. Sozuer, J. W. Haus, and R. Inguva, Phys. Rev. B 45, 13962 (1992); J. Opt. Soc. Am. B 10, 296 (1993).

${ }^{7}$ P. R. Villeneuve and M. Piche, Phys. Rev. B 46, 4969 (1992); 46, 4973 (1992).

${ }^{8}$ R. D. Meade, K. D. Brommer, A. M. Rappe, and J. D. Joannopoulos, Appl. Phys. Lett. 61, 495 (1992).

${ }^{9}$ M. Plihal, A. Shambrook, A. A. Maradudin, and P. Sheng, Opt. Commun. 80, 199 (1991); M. Plihal and A. A. Maradudin, Phys. Rev. B 44, 8565 (1991).

${ }^{10}$ E. Yablonovitch, T. J. Gmitter, R. D. Meade, A. M. Rappe, K. D. Brommer, and J. D. Joannopoulos, Phys. Rev. Lett. 67, 3380 (1991).

${ }^{11}$ R. D. Meade, K. D. Brommer, A. M. Rappe, and J. D. Joannopoulos, Phys. Rev. B 44, 13772 (1991).

${ }^{12}$ S. L. McCall, P. M. Platzman, R. Dalichaouch, D. Smith, and S. Schultz, Phys. Rev. Lett. 67, 2017 (1991).

${ }^{13}$ W. Robertson, G. Arjavalingan, R. D. Meade, K. D. Brommer, A. M. Rappe, and J. D. Joannopoulos, Phys. Rev. Lett. 68, 2023 (1992).

14J. B. Pendry and A. MacKinnon, Phys. Rev. Lett. 69, 2772
(1992); J. B. Pendry, J. Mod. Opt. 41, 209 (1994).

${ }^{15}$ M. M. Sigalas, C. M. Soukoulis, E. N. Economou, C. T. Chan, and K. M. Ho, Phys. Rev. B 48, 14121 (1993).

${ }^{16}$ K. M. Ho, C. T. Chan, C. M. Soukoulis, R. Biswas, and M. Sigalas, Solid State Commun. 89, 413 (1994).

${ }^{17}$ E. Ozbay, A. Abeyta, G. Tuttle, M. Tringides, R. Biswas, C. M. Soukoulis, C. T. Chan, and K. M. Ho, Phys. Rev. B 50, 1945 (1994).

${ }^{18}$ E. Ozbay, E. Michel, G. Tuttle, M. Sigalas, R. Biswas, and K. M. Ho, Appl. Phys. Lett. 64, 2059 (1994).

${ }^{19}$ E. Ozbay, E. Michel, G. Tuttle, R. Biswas, K. M. Ho, J. Bostak, and D. M. Bloom, Opt. Lett. 19, 1155 (1994).

${ }^{20}$ M. M. Sigalas, C. M. Soukoulis, C. T. Chan, and K. M. Ho, Phys. Rev. B 49, 11080 (1994).

${ }^{21}$ A. R. McGurn and A. A. Maradudin, Phys. Rev. B 48, 17576 (1993).

${ }^{22}$ D. R. Smith, S. Shultz, N. Kroll, M. Sigalas, K. M. Ho, and C. M. Soukoulis, Appl. Phys. Lett. 65, 645 (1994).

${ }^{23}$ J. D. Jackson, Classical Electrodynamics (Wiley, New York, 1975).

${ }^{24}$ Electromagnetic Theory of Gratings, edited by R. Pettit (Springer-Verlag, Berlin, 1980).

${ }^{25}$ W. Lamb, D. M. Wood, and N. W. Ashcroft, Phys. Rev. B 21, 2248 (1980).

${ }^{26}$ E. N. Economou and M. M. Sigalas, Phys. Rev. B 48, 13434 (1993).

${ }^{27}$ N. Stefanou and A. Modinos, J. Phys. Condens. Matter 3, 8135 (1991).

${ }^{28}$ H. S. Sozuer and J. W. Haus, J. Opt. Soc. Am. B 10, 296 (1993); H. S. Sozuer and J. P. Dowling, J. Mod. Opt. 41, 231 (1994). 